\title{
Special Topic on Molecular Functional Materials and Applications
}

\author{
Daoben Zhu, Deqing Zhang \& Yunqi Liu \\ Institute of Chemistry, Chinese Academy of Sciences, Beijing 100190, China
}

Molecular functional materials have received increasing attentions in recent years because of their promising applications in various areas. Chinese scientists have made important contributions to the development of this area. In order to further promote research activities of this area in China and strengthen academic exchanges and collaborations, the Committee of Organic Solids of Chinese Chemical Society (CCS) organized the 10th National Symposium on Electronic Process in Organic Solids during August 7-10, 2015 at Peking University.

Above 100 scientists were invited to give plenary lectures and invited talks to introduce their recent research results and discuss the perspective on topics including design and synthesis of novel conjugated molecules, molecular nanostructures, optoelectronic functions of molecular materials, carbon-based materials, theoretical studies of electron transfer process, organic solar cells, and biological sensors. After discussion with editors of Sci. China Chem., the organizing committee decided to publish a special topic on "Molecular Functional Materials and Applications" to reflect the recent progresses and future perspective of this area.

This special topic includes 2 review articles and 7 re- search papers. The reviews comment on the recent advances in perovskite materials for solar cells, efficient light emitting diodes and flexible and stretchable electrodes for next generation polymer electronics. The research articles embrace the preparation of highly conducting carbon electrodes from pyrolyzation of spin-coated polyacrylonitrile thin films, the effect on molecular planarity to achieve organic ternary memory when taking triphenylamine as the spacer, the fast growth of graphene on $\mathrm{SiO}_{2} / \mathrm{Si}$ substrates by atmospheric pressure chemical vapor deposition with floating metal catalysts, as well as the synthesis and properties of some novel functional molecules and materials for organic thin-film transistors, organic light-emitting diodes and supercapacitors.

We wish the special topic can provide valuable information to the related researchers and play an active role in promoting the research work on organic solids. With its successful publication, we would like to express our special gratitude to all the authors for their scientific contributions and the referees for their critical comments. We would also like to thank Dr. Xuemei Zhang, from the editorial office of Sci. China Chem. for her help in dealing with the manuscript and organizing this topic.

*Corresponding authors: (email: zhudb@iccas.ac.cn, dqzhang@iccas.ac.cn, liuyq@iccas.ac.cn) 


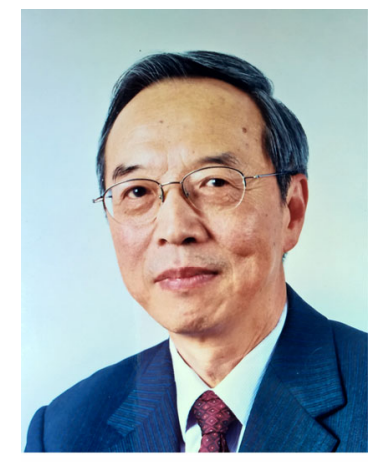

Prof. Daoben Zhu was born in Shanghai. He obtained his graduate degree from the East China University of Science and Technology in 1968. From 1977 to 1979 and 1985 to 1986, as a visiting-scholar and visiting-scientist he performed research in the Max-Planck Institute for Medical Research in Heidelberg, Germany, respectively. He was the vice-president of National Natural Science Foundation of China (NSFC) from 2000-2007, president of Chinese Chemical Society, and vice-president of Chinese Materials Society. Currently, He is a professor and Director of the Organic Solids Laboratory, Institute of Chemistry, Chinese Academy of Sciences, and a fellow of Asia-Pacific Academy of Materials, Royal Society of Chemistry (UK) and honor member of Korean Chemical Society. He was selected as an academician of Chinese Academy of Sciences in 1997, and the member of the Third World Academy of Sciences (TWAS) in 2009. His research interests include molecular materials and devices. He received second-class prizes of National Natural Sciences of China in 1988, 2002, 2004, 2007 and 2014, respectively, and Tan Kah Kee Science Award in chemistry in 2012.

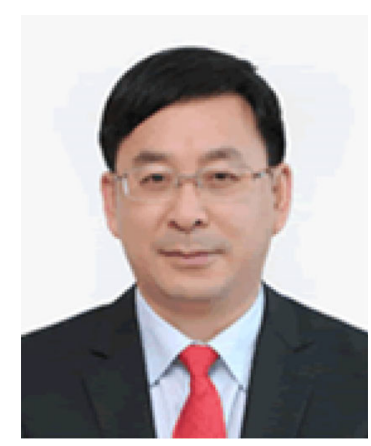

Prof. Deqing Zhang studied chemistry in Beijing Normal University from 1983 to 1987 . He then joined in the Institute of Chemistry, Chinese Academy of Sciences (ICCAS) as a graduate student and obtained his MS. degree in Organic Chemistry in 1990. He conducted research on electron donor-acceptor cyclophanes at the Max-Planck Institute for Medical Research in Heidelberg (Germany) under the supervision of Prof. H. A. Staab, and received his $\mathrm{PhD}$ degree from Ruprecht-Karls University Heidelberg in 1996. He is now a research Professor in Institute of Chemistry, Chinese Academy of Sciences (ICCAS) and the Director of the institute. His research interests include development of external stimuli-responsive molecular systems for molecular switches, logic gates and chemical/bio-sensors. He also shows interests in design and synthesis of organic functional materials and organic nanoassemblies.

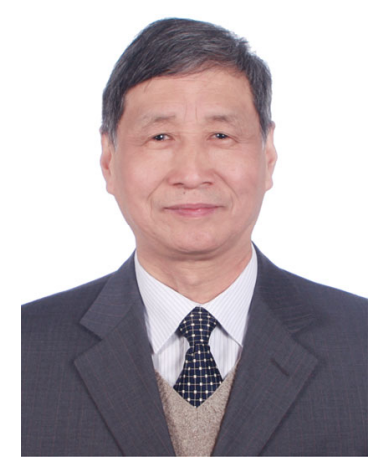

Prof. Yunqi Liu was born in Jiangsu in 1949, graduated from Nanjing University in 1975, received a doctorate from Tokyo Institute of Technology, Japan in 1991. Presently, he is a Professor at the Institute of Chemistry, Chinese Academy of Sciences (CAS). He was elected as an Academician of CAS in 2015. His current research interests include design and synthesis of molecular materials, including $\pi$-conjugated small molecules, polymers, and graphene, fabrication of related devices, including field-effect transistors and molecular electronics, and investigation of their electronic properties. 